\title{
Comparison of $250 \mathrm{MHz}$ electron spin echo and continuous wave oxygen EPR imaging methods for in vivo applications
}

\author{
Boris Epel, ${ }^{\text {a),b) }}$ Subramanian V. Sundramoorthy, Eugene D. Barth, \\ Colin Mailer, and Howard J. Halpern ${ }^{\mathrm{a}), \mathrm{c})}$ \\ Department of Radiation and Cellular Oncology, Center for EPR Imaging In Vivo Physiology, \\ University of Chicago, MC1105, 5841 South Maryland Avenue, Chicago, Illinois 60637
}

(Received 6 August 2010; revised 7 December 2010; accepted for publication 25 January 2011; published 22 March 2011)

\begin{abstract}
Purpose: The authors compare two electron paramagnetic resonance imaging modalities at 250 $\mathrm{MHz}$ to determine advantages and disadvantages of those modalities for in vivo oxygen imaging. Methods: Electron spin echo (ESE) and continuous wave (CW) methodologies were used to obtain three-dimensional images of a narrow linewidth, water soluble, nontoxic oxygen-sensitive trityl molecule OX063 in vitro and in vivo. The authors also examined sequential images obtained from the same animal injected intravenously with trityl spin probe to determine temporal stability of methodologies.

Results: A study of phantoms with different oxygen concentrations revealed a threefold advantage of the ESE methodology in terms of reduced imaging time and more precise oxygen resolution for samples with less than 70 torr oxygen partial pressure. Above $\sim 100$ torr, CW performed better. The images produced by both methodologies showed $p \mathrm{O}_{2}$ distributions with similar mean values. However, ESE images demonstrated superior performance in low $p \mathrm{O}_{2}$ regions while missing voxels in high $p \mathrm{O}_{2}$ regions.

Conclusions: ESE and CW have different areas of applicability. ESE is superior for hypoxia studies in tumors. (C) 2011 American Association of Physicists in Medicine.
\end{abstract}

[DOI: 10.1118/1.3555297]

Key words: electron paramagnetic resonance, oxymetry, hypoxia

\section{INTRODUCTION}

The oxygenation of a tumor has a strong impact on tumor physiology and tumor cure probability. ${ }^{1-5}$ Electron paramagnetic resonance oxygen imaging (EPROI) is a technique capable of noninvasively measuring spatial distributions of oxygen tension $\left(\mathrm{pO}_{2}\right)$ in tissues of live animals. ${ }^{6-8}$ One of the promising strategies for EPR oxymetry is based on spectroscopic imaging of a water soluble, nontoxic, oxygensensitive trityl spin probe ${ }^{9}$ injected intravenously into an animal.

At present, the most widespread EPROI technique for in vivo imaging is continuous wave $(\mathrm{CW})$ EPROI..$^{6-8,10,11} \mathrm{CW}$ EPROI has relatively simple equipment requirements and can image a large variety of spin probes. The key methodology is the spectral-spatial imaging that determines an EPR spectrum in every point of space. ${ }^{12}$ Filtered backprojection (FBP) algorithms are used for reconstruction of images with up to three spatial dimensions and one spectral dimension. ${ }^{13}$ The $p \mathrm{O}_{2}$ is measured from the linear relation between $p \mathrm{O}_{2}$ and the Lorentzian spin-packet linewidth (LW) of a spin probe ${ }^{11}$ determined by precise modeling of the EPR line shape. ${ }^{14}$ The disadvantage of $\mathrm{CW}$ imaging is its relatively slow acquisition speed because an EPR spectrum requires the magnetic field to be swept. Any acceleration of the acquisition increases the requirements on compliance of the power supply driving the magnetic field coils. Previously, we have used linear power supplies; however, in this article, we demonstrate that $\mathrm{CW}$ images can be acquired more quickly using a high compliance switching-mode power supply.

Another way to accelerate image acquisition is to use pulse methods. Pulse EPR methodologies used in vivo oxygen imaging includes single point imaging (SPI) ${ }^{15,16}$ and electron spin echo (ESE) imaging (ESEI) developed in our group. ${ }^{17}$ SPI utilizes phase encoding, while ESEI is based on frequency encoding of spatial information. Both methodologies demonstrate very high acquisition speed and $p \mathrm{O}_{2}$ fidelity. Applying our ESEI methodology to phantoms we have demonstrated that one can obtain $p \mathrm{O}_{2}$ images in a few minutes with precision better than 1 torr. $^{18}$ The ESE is detected using a two-pulse sequence. A series of three-dimensional (3D) images with different delays $(\tau)$ between $\pi / 2$ - and $\pi$-pulses is acquired and the decay of intensity in every spatial point is fitted to an exponential function. The inverse spin-spin relaxation time determined from this procedure $\left(1 / T_{2 e}\right)$ can be converted to spin-packet line full width at half height (a homogeneous EPR line broadening component) using $\mathrm{LW}=\left(2 \pi \gamma T_{2 e}\right)^{-1}$, where $\gamma$ is the gyromagnetic ratio of the electron. Unlike spectral-spatial imaging, 3D imaging does not determine the complete EPR spectrum of the spin probe. ${ }^{18}$ Instead, only relaxation time and EPR line amplitude are determined in every image voxel. Nevertheless, this information is sufficient for $p \mathrm{O}_{2}$ determination. This direct measurement of spin-spin relaxation is the major advantage of ESEI and the reason for the higher precision of $p \mathrm{O}_{2}$ determination as compared to other EPROI methods. CW imaging produces a spectrum at each spatial point, a four- 
dimensional image. For generation of spectral information, at each gradient spatial angle, a sequence of projections with different gradient amplitudes has to be obtained. For ESE imaging, a much more limited number of echoes are obtained at each spatial projection, creating a rapidly acquired sequence of 3D images. A more theoretical advantage of the pulse technique relative to the $\mathrm{CW}$ technique lies in the intrinsic dimensionality of the images. The pulse images are constructed from a sequence of 3D images obtained at specific echo times. The $\mathrm{CW}$ image is $4 \mathrm{D}$, involving the three spatial dimensions and a frequency or field dimension. ${ }^{12,19}$ It is a well-known property of the Radon transform that it is not a local transformation in even dimensions. ${ }^{20}$ That nonlocality makes it more susceptible to projection truncation artifacts.

Although CW EPROI and ESEI determine the same parameters, their imaging protocols, instrumentation, and data processing are very different. Consequently, both methods may have different responses to experimental conditions. The early comparison of CW and FID detected EPR was published in $2002 .{ }^{22}$ The substantial improvement of pulse EPR techniques and equipment since then necessitates another comparison. In previous publications, using phantoms, we demonstrated that ESEI delivers results essentially identical to CW EPROI in a shorter time. ${ }^{17,18}$ The spatial resolution of both methodologies is about $1.5 \mathrm{~mm}$ and oxygen resolution in phantoms is $\sim 3$ and $\sim 1$ torr for CW EPROI and ESEI, respectively. ${ }^{18,23}$ Living organisms present a more serious challenge for imaging. Sharp gradients of oxygen and spin-probe concentrations may be present within a single voxel of an image. The result will be an average of the voxel $p \mathrm{O}_{2}$, potentially methodology dependent. Furthermore, continuous changes of spin-probe concentration and $p \mathrm{O}_{2}$ during the experiment are to be expected. Not all these factors can be investigated using phantoms and therefore there is a need for direct in vivo intermodality comparison. In this work, we present such a comparison of the ESEI and CW EPROI modalities obtained on the same live animals using our 250 pulse $\mathrm{MHz} \mathrm{CW} /$ pulse EPR instrument.

\section{MATERIALS AND METHODS}

\section{II.A. Spin probe}

The spin probe used for the EPR imaging was a OX063 radical methyl-tris[8-carboxy-2,2,6,6-tetrakis[2hydroxyethyl]benzo[1,2-d:4, 5-d' ]bis[1,3]dithiol-4-yl]trisodium salt, molecular weight $=1427$, from GE Healthcare (Little Chalfont, Buckinghamshire, United Kingdom). A 70.0 mM OX063 solution was prepared in distilled water and then passed through a $5 \mu \mathrm{m}$ filter to remove particles large enough to occlude a capillary.

\section{II.B. Tumor model and animal protocol}

Approximately $10^{6} \mathrm{FSa}$ fibrosarcoma cells were injected intramuscularly into the right gastrocnemius muscle of 6-8 week old female $\mathrm{C} 3 \mathrm{H} / \mathrm{HeN}$ mice (Harlan Sprague-Dawley, Indianapolis, IN). These tumors grow to approximately 8-10 $\mathrm{mm}$ mean dimension in 7-10 days. During the imaging preparations and actual imaging, the mouse inhaled a $1.0 \%-$ $2.0 \%$ isoflurane atmosphere. While subjected to isoflurane anesthesia, the mouse's rectal temperature and its surface abdominal temperature were monitored at different times. IR radiant heating and contact heating by Deltaphase Isothermal Pad heating pads (Braintree Scientific, Inc., Braintree, MA) were adjusted to maintain the mouse's core temperature within the range $36-38{ }^{\circ} \mathrm{C}$. For administration of the OX063 spin probe, the mouse's tail vein was catheterized with $24 \mathrm{Ga}$ angiocatheter. To eliminate the spin probe from the bladder, we cannulated the mouse's urethra with a $20 \mathrm{Ga}$ angiocatheter whose lumen contains an internal polyethylene tube. The double lumen extended into the mouse's bladder. Water, through the internal tube, allowed flushing the mouse bladder at a rate of $20 \mathrm{ml} / \mathrm{h}$. The effluent containing the OX063 was collected for recycling well away from the imaging apparatus in order to minimize image artifacts. ${ }^{24}$ The tumor was immobilized in the resonator by a cast of vinyl polysiloxane dental impression material (GC Dental Products, Kasugai, Japan). It has been shown previously that the cast does not affect image quality. ${ }^{25}$

The complete experiment, including the animal preparation, could be up to $3 \mathrm{~h}$ long. We carefully maintained a steady level of anesthesia and monitored the breathing rate and temperature during the experiment to ensure that the oxygenation level of the tissues was similar for all measurements. Animals tolerated the procedure remarkably well.

All animal experiments were done according to the USPHS "Policy on Humane Care and Use of Laboratory Animals," and the protocols were approved by the University Of Chicago Institutional Animal Care and Use Committee (ACUP No. 69681). The University Of Chicago Animal Resources Center is an Association for Assessment and Accreditation of Laboratory Animal Care-approved animal care facility.

\section{II.C. Animal imaging protocol}

At the start of imaging, $200 \mu \mathrm{l}$ of the $70 \mathrm{mM} \mathrm{OX063 \textrm {H }}$ solution was injected into a $25 \mathrm{~g}$ animal $(1.4 \mathrm{mmol} / \mathrm{kg}$ bodyweight), which corresponds to one-sixth of the $\mathrm{LD}_{50}$ for the spin probe. ${ }^{26}$ The 280 mOsm osmolarity of the solution is near physiologic and has little effect on the animal physiology; the renal activity of an animal efficiently clears the spin probe from the blood with a half-life of a few minutes but the clearance half-life from tumor tissue is much slower ( $\sim 30 \mathrm{~min}$ ). To compensate for the clearance, a continuous infusion of the same solution with the rate of $\sim 0.22 \mathrm{ml} / \mathrm{h}$ was maintained during the experiment. The rate of the infusion was adjusted to maintain a constant trityl EPR signal level in the tumor bearing leg. Consecutive images utilizing the same modality followed each other immediately. When a modality change was desired, the change-over time was about 5 min.

\section{II.D. CW imaging}

In our previous work, ${ }^{5,18}$ the B-MN $3 \mathrm{x} \pm 40 / 60 \mathrm{C} 5$ (Bruker BioSpin Corporation, Billerica, MA), a bipolar linear mag- 
TABLE I. CW and ESE imaging protocols. For clarity, the duration of protocols is given after the protocol name (i.e., 11 min $\mathrm{CW}$ image is abbreviated as $\mathrm{CW}-11)$.

\begin{tabular}{|c|c|}
\hline Protocols & Description \\
\hline CW-11, CW-30 & $\begin{array}{l}\text { Equal solid spatial angle gradient scheme; } 66 \text { spatial } \\
\text { directions and } 14 \text { spectral angles (gradient magnitudes) } \\
\text { in the range }(-\pi / 2, \pi / 2),|\vec{G}|_{\max }=30 \mathrm{mT} / \mathrm{m} \text {; overall } 924 \\
\text { projections; spatial field of view } 3.0 \mathrm{~cm} \text {; spectral field of } \\
\text { view } 0.1 \mathrm{mT} \text {. The sweep rate during CW-11 imaging was } \\
\text { three times higher than that for } \mathrm{CW}-30 \text {. Imaging time } \\
11 \text { min for } \mathrm{CW}-11 \text { and } 30 \text { min for } \mathrm{CW}-30 \text {. }\end{array}$ \\
\hline ESE-10 & $\begin{array}{l}\text { Equal solid spatial angle gradient scheme; } 208 \\
\text { projections; }|\vec{G}|=15 \mathrm{mT} / \mathrm{m} \text {; field of view } 4.24 \mathrm{~cm} \text {; } \\
\text { baseline acquired every fourth trace (overall } 53 \text { traces); } \\
35 \mathrm{~ns} \pi / 2 \text { and } \pi \text { RF pulses, } 16 \text {-step phase cycling, } \\
40000 \text { acquisitions, including phase cycling; five images } \\
\text { with different } \tau \text { logarithmically spaced between } 0.63 \mu \mathrm{s} \\
\text { and } 2.4 \mu \mathrm{s} \text {. Imaging time } 10 \text { min. }\end{array}$ \\
\hline
\end{tabular}

netic field power supply, was used in the imager. For this work, we substituted a switching-mode power supply model 262 (Copley, Canton, MA) with considerably higher compliance $(300 \mathrm{~V}$ vs $40 \mathrm{~V}$ of B-MN supply). This allowed us to triple the maximum rate of linear magnetic field sweeping. This rate is considerably slower than the rates used for rotating gradient methodology, ${ }^{27}$ but is sufficient to demonstrate the effect of accelerated image acquisition on image signal to noise ratio (SNR). New control software and hardware with minimum programming and communication delays reduced the acquisition time for CW imaging by $35 \%$. A $19 \mathrm{~mm}$ inner diameter split top resonator was used for imaging. This resonator has a considerably lower empty $Q(\sim 150)$ than resonators dedicated solely for $\mathrm{CW}$ imaging $(\sim 300)$, but is better suited for cross-methodological comparisons. The $Q$ of the resonator loaded with a phantom was 140 . The $Q$ of the resonator with an animal was 80-120, similar to loaded $Q$ of resonators dedicated for $\mathrm{CW}$ imaging. CW EPR oxygen images were acquired and reconstructed using standard spectral-spatial FBP algorithms. ${ }^{12,13,28,29}$ Two protocols for CW acquisition with different magnetic sweep rates were used (Table I). The duration of the slower protocol, similar to that used in our previous studies, was 30 min per image, while the duration of the accelerated protocol was 11 min per image. The number of projections and amplitudes of gradients were kept identical. Each EPR projection spectrum was acquired with 256 points. The spectrum was then subjected to a four-point running average filter with Gaussian weights and then subsampled to $256 / 4=64$ points. Projections were filtered with a 3D Ram-Lak filter with a cutoff at 0.5 times the Nyquist frequency and four times linearly interpolated. $^{28,30}$ The fitting algorithm of Robinson et al. ${ }^{14}$ was used to extract the spin-packet line width and signal amplitude from the spectral dimension of each spatial voxel of the image. All data processing was performed using inhouse MATLAB (The Mathworks, Inc., Natick, MA) scripts.

\section{II.E. Electron spin echo imaging}

In this work, we used the versatile pulse $250 \mathrm{MHz}$ imager described in detail elsewhere. ${ }^{18}$ To utilize the full power of our $2 \mathrm{~kW}$ RF amplifier ${ }^{31}$ (Tomco Technologies, Norwood SA, Australia), the transmit-receive switch of the imager was redesigned utilizing a new protection scheme and high power components. $^{32}$ A pulse amplitude modulation switch was added to produce $\pi / 2$ - and $\pi$-pulses of equal duration. ${ }^{33}$ The imager control software SPECMAN4EPR version 1.1.5 (Ref. 34) that makes possible nonstop multiple image acquisition was used. The same $19 \mathrm{~mm}$ inner diameter split top resonator was used for CW and ESE imaging. For pulse experiments, the $Q$ was reduced to $13-14$ using a $750 \Omega$ shunt resistor. ${ }^{35}$

ESE image data collection uses a 3D FBP protocol. ${ }^{13,17}$ An equal solid angle scheme ${ }^{28}$ was used for gradient directional spacing. To reduce FBP reconstruction artifacts, the acquired set of projections was fourfold linearly interpolated $^{30}$ and filtered with a 3D Ram-Lak filter with a cutoff at 0.5 times the Nyquist frequency. The data acquisition and processing methods are discussed in detail elsewhere. ${ }^{18}$ A summary of the protocol is given in Table I.

The electron spin echoes were generated with a $\pi / 2-\tau-\pi-\tau$ echo pulse sequence. We used $35 \mathrm{~ns} \pi / 2$ - and $\pi$-pulses that had $6 \mathrm{~dB}$ differences in power. The equal bandwidth of the pulses produces uniform excitation of spins and helps to reduce the reflections from the resonator, whose loaded quality factor $Q \sim 14$ for phantoms and $Q$ $=13.2-13.8$ for mice was chosen to match the bandwidth of the sequence. An applied RF power of $52 \mathrm{dBm}$ for the $\pi$-pulse maximized the echo height. To obtain image projections, the echoes were Fourier transformed. To avoid truncation artifacts due to the dead time of the imager, the analysis involved only the $4 \mu$ s following the echo maximum. We kept in subsequent images only those voxels with signal amplitude greater than $15 \%$ of the voxel with the maximum amplitude in the image taken at the earliest $\tau$. This threshold level selects an image volume comparable to that of high resolution imaging techniques such as CT or MRI. All data processing was performed using in-house MATLAB (The Mathworks, Inc., Natick, MA) scripts.

\section{II.F. Phantom imaging}

The spin probe was contained in a flat-bottomed borosilicate glass cylinder of $9.5 \mathrm{~mm}$ inner diameter and $45 \mathrm{~mm}$ length. The trityl samples were bubbled with calibrated water-saturated gas mixtures of oxygen and nitrogen until equilibrated and then sealed with epoxy. During measurements, samples were placed into the resonator horizontally along the resonator's axis of symmetry and centered in the axial plane of the resonator. For statistics, only those voxels inside resonator volume were analyzed. The image comparisons were restricted to voxels located in both images. The difference between selections of voxels in images did not exceed $2 \%$ of the overall voxels for all pairs and the majority of nonmatched voxels were localized on the periphery of images. 
TABLE II. Comparison of $p \mathrm{O}_{2}$ precision as determined from a single phantom image (method M1) and from consequent phantom images (method M2). The error of $\operatorname{std}(\Delta)$ is below $20 \%$ of the presented values.

\begin{tabular}{|c|c|c|c|c|c|c|}
\hline \multirow[b]{2}{*}{$\begin{array}{l}\mathrm{pO}_{2} \\
\text { (torr) }\end{array}$} & \multicolumn{3}{|c|}{ Method M1 } & \multicolumn{3}{|c|}{ Method M2 } \\
\hline & $\begin{array}{l}\text { CW-30, std } \\
\text { (torr) }\end{array}$ & $\begin{array}{l}\text { CW-11, std } \\
\text { (torr) }\end{array}$ & $\begin{array}{l}\text { ESE-10, std } \\
\quad \text { (torr) }\end{array}$ & $\begin{array}{l}\text { CW-30, } \operatorname{std}(\Delta) / \sqrt{ } 2 \\
\text { (torr) }\end{array}$ & $\begin{array}{l}\text { CW-11, } \operatorname{std}(\Delta) / \sqrt{ } 2 \\
\text { (torr) }\end{array}$ & $\begin{array}{c}\text { ESE-10, } \operatorname{std}(\Delta) / \sqrt{ } 2 \\
\text { (torr) }\end{array}$ \\
\hline 0 & 4.0 & 10.0 & 0.7 & 3.3 & 8.0 & 0.6 \\
\hline 60 & 4.3 & 11.7 & 3.5 & 3.5 & 8.4 & 2.8 \\
\hline 80 & 4.7 & 14.2 & 5.1 & 3.0 & 13.1 & 4.2 \\
\hline 140 & 9.5 & 14.4 & 22.0 & 9.0 & 12.0 & 20.9 \\
\hline
\end{tabular}

\section{II.G. Calibration}

The OX063H spin-packet LW and oxygen partial pressure are linearly related with a coefficient equal to 18.4 torr $/ \mu \mathrm{T}$. The $\mathrm{OX} 063 \mathrm{H}$ spin-packet linewidth at 0 torr oxygen and animal temperature $\left(37^{\circ} \mathrm{C}\right)$ is equal to $1.2 \mu \mathrm{T}$. For $\mathrm{CW}$, several additional factors contribute to the 0 torr linewidth broadening, ${ }^{11}$ resulting in the spin-packet linewidth of $1.8 \mu \mathrm{T}$. The intensities of the images were calibrated from the intensity of the $1 \mathrm{mM}$ deoxygenated OX063H homogeneous phantom. This established an average voxel concentration, the number of spins in each voxel of an image. For ESEI calibration, the image formed by extrapolating voxel intensity to echo time zero was used.

\section{II.H. Image registration}

ESE oxygen images have little anatomic information. In order to discriminate tumor from other tissues in our ESE images, we must make an anatomic MRI image and register it to the ESE image. ${ }^{36}$ In both images, we locate a set of fiducial tubes that bear a fixed spatial relationship to the mouse leg. These fiducials are embedded in the same vinyl polysiloxane dental material cast holding the leg. ${ }^{25}$ This defines a coordinate transformation from the MRI image that allows us to import anatomic information, especially the tumor boundary, to the ESE image.

\section{RESULTS}

The comparison of live animal images obtained using different methodologies is not straightforward. Neither CW or ESE can be considered as a "gold standard" of 3D noninvasive oxymetry. ${ }^{37}$ In our previous studies, ${ }^{18,23}$ we evaluated the $\mathrm{O}_{2}$ precision from an image of a deoxygenated phantom by calculating the standard deviation of the $\mathrm{pO}_{2}$ of image voxels with amplitudes above a certain threshold $(15 \%$ of maximum). We will call this method Ml. On a live animal, there is no predefined knowledge of $\mathrm{pO}_{2}$. Moreover, $\mathrm{pO}_{2}$ both in tumors and normal tissues changes with time. ${ }^{38}$ Therefore, method Ml cannot be applied with any accuracy. For in vivo comparisons, we have used another method (M2), in which we take sequential images of the same object and then calculate statistics for the voxel-by-voxel $\mathrm{pO}_{2}$ difference between those images. Assuming that image noise is not spatially correlated, the standard deviations calculated using method M2 will be larger by a factor of $\sqrt{2}$ than those calculated using method $\mathrm{Ml}$ when applicable.

Table II demonstrates that methods $\mathrm{Ml}$ and $\mathrm{M} 2$ are indeed give similar results on $1 \mathrm{mM} \mathrm{OX63H}$ phantoms. To cover the range of physiologically relevant $\mathrm{pO}_{2}$ values, we studied four phantoms with $\mathrm{pO}_{2}$ from 0 to 140 torr. The precision of CW-30 measurements is lower than reported previously (3.0 torr) (Ref. 23) due to twice lower $Q$ of the resonator used in this work. One immediate result is that the ESE method shows a much stronger decline in the $\mathrm{pO}_{2}$ precision compared to $\mathrm{CW}$ for phantoms with high $\mathrm{pO}_{2}$. The precision of $p \mathrm{O}_{2}$ determination is generally expected to be lower for high $p \mathrm{O}_{2}$ phantoms due to decreases in SNR associated with the EPR line broadening in case of $\mathrm{CW}$ or accelerated $T_{2 e}$ relaxation in the ESE case. The SNR of the pulse methodology is largely governed by the dead time of the imager. This dead time determines the shortest delay between pulses in the ESE pulse sequence, for which the echo can be obtained (550 ns for animal imaging in our case). For high $\mathrm{pO}_{2}$ samples, a considerable part of spin system magnetization has already relaxed during this delay, resulting in a poorer image SNR (see Table III). According to the Table II, the ESEI precision of $p \mathrm{O}_{2}$ determination is superior to the precision of the CW-30 (30 min CW) $p \mathrm{O}_{2}$ imaging up to $\sim 70$ torr. The $\mathrm{CW}-11$ images, which have durations similar to ESE images, have larger $\mathrm{pO}_{2}$ standard deviations for $\mathrm{pO}_{2}$ values below $\sim 100$ torr. However, they do determine the correct mean $p \mathrm{O}_{2}$ values and can be used for quick assessment of oxygenation in qualitative studies.

We performed a comparison of the CW and ESE imaging methodologies on a number of mice. Each comparison was

TABLE III. SNR of images recorded on phantoms with different $p \mathrm{O}_{2}$. SNR is determined as the ratio of average signal intensity at the center of a phantom and standard deviation of amplitude far from a phantom. For CW, SNR is measured for a 3D slice of 4D image corresponding to the point of maximum intensity in EPR spectrum. For ESE, SNR is measured for 3D image recorded at shortest $\tau$. The error of SNR is below $5 \%$ of the presented values.

\begin{tabular}{cccc}
\hline \hline \multirow{2}{*}{$\begin{array}{c}3 \\
\text { (torr) }\end{array}$} & $\mathrm{CW}-30$ & $\mathrm{CW}-11$ & Image SNR \\
\cline { 2 - 4 } & 90 & 70 & 86 \\
0 & 88 & 69 & 60 \\
60 & 91 & 70 & 50 \\
80 & 76 & 55 & 21 \\
140 & & & \\
\hline \hline
\end{tabular}


TABLE IV. Comparison of sequential in vivo images obtained from a tumor bearing leg. The method M2 is used; mean and standard deviations of the populations of differences between the $p \mathrm{O}_{2}$ voxels of the images are calculated. For each comparison, $n=2-3$ experiments on different animals were performed, the range of results is presented.

\begin{tabular}{ccc}
\hline \hline In vivo images compared & $\begin{array}{c}\operatorname{mean}(\Delta) \\
\text { (torr) }\end{array}$ & $\begin{array}{c}\operatorname{std}(\Delta) / \sqrt{ } 2 \\
\text { (torr) }\end{array}$ \\
\hline CW-30 vs CW-30, $n=3$ & $1.0-3.0$ & $11-14$ \\
CW-11 vs CW-11, $n=2$ & $2.0-3.0$ & $29-30$ \\
CW-30 vs ESE-10, $n=2$ & $1.3-3.0$ & $11-14$ \\
ESE-10 vs ESE-10, $n=3$ & $0.9-1.1$ & $6.5-7.0$ \\
ESE-10 vs ESE-10, $n=3$ voxels below 40 torr only & 0.7 & $3.5-6.0$ \\
ESE-10 vs ESE-10, $n=3$ 40 min between images & $1.5-5.0$ & $7-11$ \\
\hline \hline
\end{tabular}

repeated on two to three different animals to reduce the effects of any differences in the physiology of the animals. The results are summarized in the Table IV. Representative slices of CW and ESE spin-probe concentration images obtained from the same animal are presented in plots a and b of Fig. 1, respectively. The CW and ESE images are shown in the same coordinate system and identical slices are selected. The average concentration of spin probe as reported by EPR was $\sim 0.4 \mathrm{mM}$ in tumor and $\sim 0.3 \mathrm{mM}$ in the animal body. The corresponding slices of the $p \mathrm{O}_{2}$ images are presented in the columns [Fig. 1(d) (CW) and Fig. 1(e) (ESE)]. From the figure, it is readily apparent that the spatial outlines and general features of the ESE and CW images are very similar. The general impression shown by the images is that the $\mathrm{CW}$ images show lower $p \mathrm{O}_{2}$ values than the ESE. However, the frequency histograms of the images (Fig. 2) are similar. For the histograms, all fitted voxels of the three-dimensional images were analyzed. One can notice differences between CW and ESE histograms in the very low and very high $p \mathrm{O}_{2}$ areas. The low $p \mathrm{O}_{2}$ wing of the $\mathrm{CW}$ histogram extends to negative values and demonstrates higher level of $p \mathrm{O}_{2}$ uncertainty than in the ESE image (no negative values are shown in the histogram). This correlates with phantom results. The high $p \mathrm{O}_{2}$ side of the $\mathrm{CW}$ histogram is populated slightly more than the ESE histogram. This effect is because of the exclusion of voxels with $p \mathrm{O}_{2}$ above $\sim 70$ torr from the fitting mask of ESE images due to their very low intensity and SNR.

Using the M2 method, we evaluated the $p \mathrm{O}_{2}$ precision for consecutive CW and ESE images obtained from the same animal. The range of values obtained in multiple experiments is presented in the Table IV. The standard deviation between CW EPR images was found to be larger than the deviation between ESE images. Because the ESE-10 imaging is three times faster than the CW-30 imaging, we performed an experiment to establish the long term stability of $p \mathrm{O}_{2}$ in an animal. The comparison of the ESE-10 images obtained on the same animal and separated by $40 \mathrm{~min}$ is presented in the Table IV. The standard deviation of voxel $p \mathrm{O}_{2}$ between the temporally adjacent pulse images appeared to be similar to those separated by $40 \mathrm{~min}$. However, over that $40 \mathrm{~min}$ time interval, we observed a decrease in the mean $p \mathrm{O}_{2}$ in the animals by $2-5$ torr. We believe that this decrease is the effect of the anesthesia on the mice.

\section{DISCUSSION}

Phantom studies (see Table II and prior works ${ }^{17,18}$ ) demonstrated very high levels of correlation between $p \mathrm{O}_{2}$ images obtained from the same phantom using CW and ESE. The differences between the $p \mathrm{O}_{2}$ from the same voxels in images
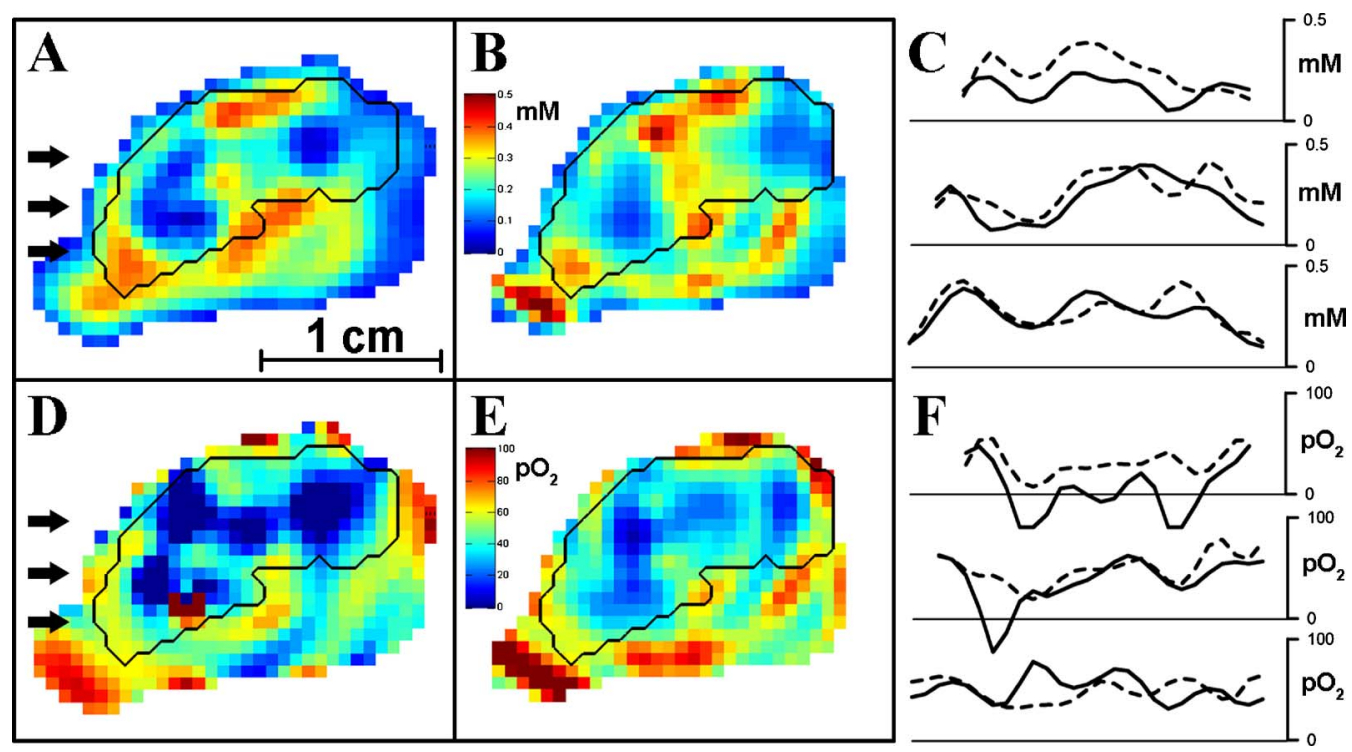

FIG. 1. Selected sagittal slices (0.66 mm thick) from three-dimensional images of tumor bearing mouse leg. First row: Spin-probe concentration images obtained using (a) CW-30 and (b) ESE-10. (c) The spin-probe concentration from images (a) (solid line) and (b) (dashed line) along the paths shown in (a) by arrows. Second row: Oxygen images obtained using (d) CW-30 and (f) ESE-10. The $p \mathrm{O}_{2}$ from images (d) (solid line) and (e) (dashed line) along the paths shown in (d) by arrows. Only voxels with amplitude above $15 \%$ of the average spin-probe concentration are shown. The tumor region obtained from a registered $T_{2}$ weighted MRUs outlined. 


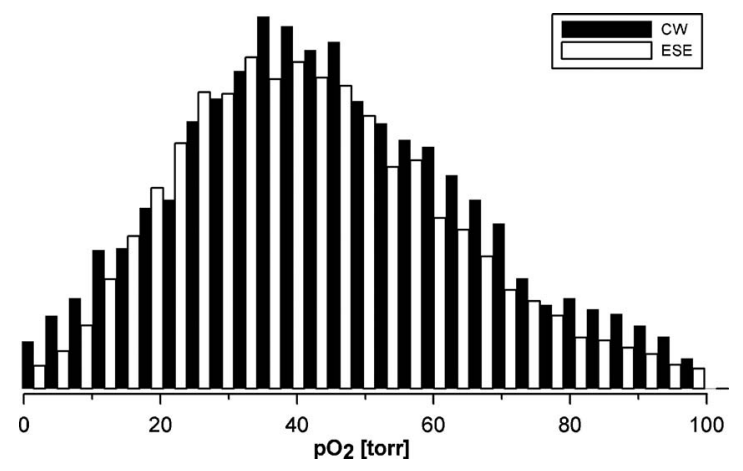

FIG. 2. Histogram of $\mathrm{pO}_{2}$ values between 0 and 100 torr obtained on a mouse leg bearing a tumor using $\mathrm{CW}$ and ESE methodologies.

obtained from live animals showed greater standard deviations. Multiple sources of error contribute to the additional $\mathrm{pO}_{2}$ standard deviation.

The $\mathrm{pO}_{2}$ in a live animal can change. ${ }^{38}$ Dewhirst et $a l{ }^{38-40}$ investigated the fluctuation of oxygen $\mathrm{pO}_{2}$ in tumor tissues and found oscillations with about 10 torr amplitude and a period of approximately $10 \mathrm{~min}$. Since the largest part of the tissue imaged is tumor (see outline in the Fig. 1), these data are applicable to our study. Hence, some of the standard deviations in CW and ESEI images can be attributed to physiologic processes. This factor, however, should affect both methodologies equally, while the one-to-one comparison of CW and ESEI images (see Table IV) demonstrates significantly lower standard deviations in the ESE images, especially in hypoxic regions which are the primary focus of studies in tumors. ${ }^{11}$

The higher resolution of the ESE technique comes at a price. With higher $p \mathrm{O}_{2}$ resolution, the influence of subtle effects on relaxation must be determined and related to $p \mathrm{O}_{2}$ effect. One of these influences is the concentration dependence of $T_{2 e}$. The literature indicates some disagreement on this subject. ${ }^{9,16}$ One of the difficulties from early work is due to the use of CW EPR to determine this effect. Even the use of either Voigt line shape analysis ${ }^{8,41}$ or the use of an accurate model of the modulation broadened spin packet cannot compensate for the relatively small fractional response of the EPR line to the effect of concentration. We believe that prior estimates of the concentration broadening effect in vivo have underestimated it. ${ }^{42}$ Moreover, imaging determines the number of spins in a given volume but the volume of distribution of the spins within an image voxel is difficult to determine due to the exclusion of trityl from intact cells. This, in turn, reduces the accuracy of the determination of the spin-probe concentration. The confounding broadening effect of spin concentration then blurs the $\mathrm{pO}_{2}$ values. In our in vivo experiments, the concentration effect was minimized by using the lowest feasible spin-probe concentration.

What are the reasons for the poorer reproducibility of the in vivo $\mathrm{CW}$ measurements? In our measurements, we compensated for the SNR advantage of pulse methods ${ }^{17,22,43}$ by taking $\mathrm{CW}$ images over a longer time. However, a greater $\mathrm{SNR}$ in the CW images did not lead to better oxygen resolution. The difference of the imaging times may be a factor by itself due to the time evolution of animal oxygenation. We ruled out this effect by taking multiple ESE images separated by a $40 \mathrm{~min}$ time interval (see Table IV). There were no appreciable differences between the several ESE images obtained over the duration of the single $\mathrm{CW}$ image acquisition. Another factor that can affect reproducibility of in vivo $\mathrm{CW}$ images might be the experimental hardware. CW measurements are done in a resonator with quality factor $Q$ of 80 150 , up to ten times greater than in the pulsed system, $Q$ $\sim 14$. This makes the $\mathrm{CW}$ resonator more sensitive to the environment and animal motion. Although the frequency drift of the resonator is compensated by the AFC unit and a field-frequency lock, the change of the resonator coupling with frequency is not compensated, potentially altering the line shape by absorption/dispersion mixing. As noted in Sec. I, an important theoretical advantage of the lower dimensionality of ESE imaging (3D compared to $4 \mathrm{D}$ for $\mathrm{CW}$ ) is that the filtered backprojection in odd dimensions is local and less susceptible to projection truncation artifacts. ${ }^{21,44}$ Here we refer to the dimensionality of inverse Radon transformation, used to produce an image, and not to the dimensionality of the final image, which will have four dimensions in both cases. The wings of the Lorentzian spin-packet line (and hence the projection) are minimally truncated by the limited magnetic field range in 4D spectral-spatial imaging used by $\mathrm{CW}$, creating, in theory at least, some artifact. Another relative advantage of the ESE method comes from the very straightforward relationship between the $T_{2 e}$ and the observed signals. In contrast, fitting of the $\mathrm{CW}$ line shape has multiple parameters and depends on our ability to measure small changes in width due to oxygen in the underlying Lorentzian line width in the face a much larger, albeit fixed, inhomogeneous linewidth component. As a result, CW EPROI may require data with greater SNR than ESEI for equal quality fits.

One of the weaknesses of the ESE methodology is its performance in high $\mathrm{pO}_{2}$ areas. The importance of this aspect of the in vivo image is experiment and application dependent. The major factor that affects precision in these areas is the imager's dead time. The dead time of our imager $(\sim 550 \mathrm{~ns})$ is one-third longer than the estimated dead time of $\sim 35^{*} \mathrm{Q} / 2 \pi \nu=312$ ns of a resonator with $Q$ of $\sim 14$ at 250 $\mathrm{MHz}$ and $52 \mathrm{dBm}$ of RF power. This difference is caused by spurious transients introduced by the use of a high power circulator. $^{32}$ We expect that further improvement of our transmit/receive switch design will decrease the dead time and enhance high $\mathrm{pO}_{2}$ ESE images. The dead time can also be reduced by decreasing $Q$ but this is less desirable due to the concomitant reduction in the image SNR.

\section{CONCLUSIONS}

A comparison of oxygen images from phantoms and animals, obtained using the trityl paramagnetic molecule as the oxygen-sensitive spin probe, demonstrated a clear similarity between CW and ESE EPROI methodologies. However, ESE images with equivalent $p \mathrm{O}_{2}$ and spatial resolution take one- 
third of the $\mathrm{CW}$ image acquisition time and give superior reproducibility in hypoxic areas, which are of greater biologic relevance for in vivo imaging of tumors. For $p \mathrm{O}_{2}$ above 70 torr, CW images show higher SNR and oxygen resolution at a price of tripled acquisition time. Only above 100 torr are $\mathrm{CW}$ images superior both in terms of acquisition time and precision.

\section{ACKNOWLEDGMENTS}

The authors acknowledge their colleagues from the Center for EPR Imaging In Vivo Physiology. Special thanks to Dr. Charles Pelizzari of the University of Chicago for helpful discussions. This work is supported by NIH Grant Nos. P41 EB002034 and R01 CA98575.

\footnotetext{
${ }^{\text {a) }}$ Authors to whom correspondence should be addressed.

b)Electronic mail: bepel@uchicago.edu; Telephone: +1(773)834-1938; Fax: +1(773)702-5940.

${ }^{c)}$ Electronic mail: h-halpern@uchicago.edu; Telephone: +1(773)7026871; Fax: +1(773)702-5940.

${ }^{1}$ G. Schwarz, "Uber Desensibilisierung gegen Rontgen-und Radiumstrahlen," Munch Med. Wochenschr 56, 1217-1218 (1909).

${ }^{2}$ T. G. Graeber, C. Osmanian, T. Jacks, D. E. Housman, C. J. Koch, S. W. Lowe, and A. J. Giaccia, "Hypoxia-mediated selection of cells with diminished apoptotic potential in solid tumours," Nature (London) 379, 88-91 (1996).

${ }^{3}$ J. M. Henk and C. W. Smith, "Radiotherapy and hyperbaric-oxygen in head and neck cancer: Interim report of second clinical trial," Lancet 310, 104-105 (1977)
}

${ }^{4}$ M. Elas, R. Bell, D. Hleihel, E. D. Barth, C. Mcfaul, C. R. Haney, J. Bielanska, K. Pustelny, K. H. Ahn, C. A. Pelizzari, M. Kocherginsky, and H. J. Halpern, "Electron paramagnetic resonance oxygen image hypoxic fraction plus radiation dose strongly correlates with tumor cure in FSA fibrosarcomas," Int. J. Radiat. Oncol., Biol., Phys. 71, 542-549 (2008).

${ }^{5}$ H. J. Halpern, M. Elas, R. Bell, D. Hleihel, E. D. Barth, C. R. Chaney, K. Ahn, C. A. Pelizzari, M. Kocherginsky, and R. R. Weichselbaum, "Electron paramagnetic resonance oxygen images correlates spatially and quantitatively with increased vascular endothelial growth factor concentrations," Int. J. Radiat. Oncol., Biol., Phys. 75, S42-S42 (2009).

${ }^{6}$ H. J. Halpern, D. P. Spencer, J. Vanpolen, M. K. Bowman, A. C. Nelson, E. M. Dowey, and B. A. Teicher, "Imaging radio-frequency electron-spinresonance spectrometer with high-resolution and sensitivity for in vivo measurements," Rev. Sci. Instrum. 60, 1040-1050 (1989).

${ }^{7}$ P. Kuppusamy, M. Chzhan, K. Vij, M. Shteynbuk, D. J. Lefer, E. Giannella, and J. L. Zweier, "3-dimensional spectral spatial EPR imaging of free-radicals in the heart-A technique for imaging tissue metabolism and oxygenation," Proc. Natl. Acad. Sci. U.S.A. 91, 3388-3392 (1994).

${ }^{8}$ H. J. Halpern, C. Yu, M. Peric, E. Barth, D. J. Grdina, and B. A. Teicher, "Oxymetry deep in tissues with low-frequency electron-paramagneticresonance," Proc. Natl. Acad. Sci. U.S.A. 91, 13047-13051 (1994).

${ }^{9}$ J. H. Ardenkjær-Larsen, I. Laursen, I. Leunbach, G. Ehnholm, L. G. Wistrand, J. S. Petersson, and K. Golman, "EPR and DNP properties of certain novel single electron contrast agents intended for oximetric imaging," J. Magn. Reson. 133, 1-12 (1998).

${ }^{10}$ L. J. Berliner and H. Fujii, "Magnetic-resonance imaging of biological specimens by electron-paramagnetic resonance of nitroxide spin labels," Science 227, 517-519 (1985).

${ }^{11}$ M. Elas, B. B. Williams, A. Parasca, C. Mailer, C. A. Pelizzari, M. A. Lewis, J. N. River, G. S. Karczmar, E. D. Barth, and H. J. Halpern, "Quantitative tumor oxymetric images from 4D electron paramagnetic resonance imaging (EPRI): Methodology and comparison with blood oxygen level-dependent (BOLD) MRI," Magn. Reson. Med. 49, 682-691 (2003).

${ }^{12}$ M. M. Maltempo, S. S. Eaton, and G. R. Eaton, "Spectral-spatial twodimensional EPR imaging," J. Magn. Reson. (1969-1992) 72, 449-455 (1987).

${ }^{13}$ G. R. Eaton, S. S. Eaton, and K. Ohno, EPR Imaging and In Vivo EPR (CRC, Boca Raton, 1991).
${ }^{14}$ C. Mailer, B. H. Robinson, B. B. Williams, and H. J. Halpern, "Spectral fitting: The extraction of crucial information from a spectrum and a spectral image," Magn. Reson. Med. 49, 1175-1180 (2003).

${ }^{15}$ S. Subramanian, N. Devasahayam, R. Murugesan, K. Yamada, J. Cook, A. Taube, J. B. Mitchell, J. A. B. Lohman, and M. C. Krishna, "Singlepoint (constant-time) imaging in radiofrequency Fourier transform electron paramagnetic resonance," Magn. Reson. Med. 48, 370-379 (2002).

${ }^{16}$ K. Matsumoto, S. Subramanian, N. Devasahayam, T. Aravalluvan, R. Murugesan, J. A. Cook, J. B. Mitchell, and M. C. Krishna, "Electron paramagnetic resonance imaging of tumor hypoxia: Enhanced spatial and temporal resolution for in vivo $\mathrm{pO}(2)$ determination," Magn. Reson. Med. 55, 1157-1163 (2006).

${ }^{17}$ C. Mailer, S. V. Sundramoorthy, C. A. Pelizzari, and H. J. Halpern, "Spin echo spectroscopic electron paramagnetic resonance imaging," Magn. Reson. Med. 55, 904-912 (2006).

${ }^{18}$ B. Epel, S. V. Sundramoorthy, C. Mailer, and H. J. Halpern, "A versatile high speed 250-MHz pulse imager for biomedical applications," Concepts Magn. Reson., Part B 33B, 163-176 (2008).

${ }^{19}$ P. C. Lauterbur, D. N. Levin, and R. B. Marr, "Theory and simulation of NMR spectroscopic imaging and field plotting by projection reconstruction involving an intrinsic frequency dimension," J. Magn. Reson. (19691992) 59, 536-541 (1984).

${ }^{20}$ S. R. Deans, The Radon Transformation and Some of Its Applications (Wiley, New York, 1983).

${ }^{21} \mathrm{X}$. C. Pan and Y. Zou, "Image reconstruction in regions of interest from truncated radon transforms of even dimensions," Inverse Probl. 21, 11691177 (2005).

${ }^{22}$ K. I. Yamada, R. Murugesan, N. Devasahayam, J. A. Cook, J. B. Mitchell, S. Subramanian, and M. C. Krishna, "Evaluation and comparison of pulsed and continuous wave radiofrequency electron paramagnetic resonance techniques for in vivo detection and imaging of free radicals," J. Magn. Reson. 154, 287-297 (2002).

${ }^{23}$ K. H. Ahn and H. J. Halpern, "Simulation of 4D spectral-spatial EPR images," J. Magn. Reson. 187, 1-9 (2007).

${ }^{24}$ C. R. Haney, A. D. Parasca, K. Ichikawa, B. B. Williams, M. Elas, C. A. Pelizzari, and H. J. Halpern, "Reduction of image artifacts in mice by bladder flushing with a novel double-lumen urethral catheter," Mol. Imaging 5, 175-179 (2006).

${ }^{25}$ C. R. Haney, X. Fan, A. D. Parasca, G. S. Karczmar, H. J. Halpern, and C. A. Pelizzari, "Immobilization using dental material casts facilitates accurate serial and multimodality small animal imaging," Concepts Magn. Reson., Part B 33B, 138-144 (2008).

${ }^{26}$ M. C. Krishna, S. English, K. Yamada, J. Yoo, R. Murugesan, N. Devasahayam, J. A. Cook, K. Golman, J. H. Ardenkjaer-Larsen, S. Subramanian, and J. B. Mitchell, "Overhauser enhanced magnetic resonance imaging for tumor oximetry: Coregistration of tumor anatomy and tissue oxygen concentration," Proc. Natl. Acad. Sci. U.S.A. 99, 2216-2221 (2002).

${ }^{27}$ S. Subramanian, J. W. Koscielniak, N. Devasahayam, R. H. Pursley, T. J. Pohida, and M. C. Krishna, "A new strategy for fast radiofrequency CW EPR imaging: Direct detection with rapid scan and rotating gradients," J. Magn. Reson. 186, 212-219 (2007).

${ }^{28}$ K. H. Ahn and H. J. Halpern, "Spatially uniform sampling in 4-D EPR spectral-spatial imaging," J. Magn. Reson. 185, 152-158 (2007).

${ }^{29}$ B. B. Williams and H. J. Halpern, "In vivo EPR imaging," in Biomedical EPR-Part A: Free Radicals, Metals, Medicine, and Physiology, edited by S. S. Eaton, G. R. Eaton, and L. J. Berliner (Kluwer Academic/Plenum Publishers, New York, 2005), Vol. 23, pp. 283-319.

${ }^{30} \mathrm{~K}$. H. Ahn and H. J. Halpern, "Comparison of local and global angular interpolation applied to spectral-spatial EPR image reconstruction," Med. Phys. 34, 1047-1052 (2007).

${ }^{31}$ R. W. Quine, G. R. Eaton, and S. Dillon, "Fast-response VHF pulsed 2 KW power amplifiers," Concepts Magn. Reson., Part B 29B, 185-190 (2006).

${ }^{32}$ S. V. Sundramoorthy, B. Epel, C. Mailer, and H. J. Halpern, "A passive dual-circulator based transmit/receive switch for use with reflection resonators in pulse electron paramagnetic resonance," Concepts Magn. Reson., Part B 35B, 133-138 (2009).

${ }^{33}$ R. W. Quine, M. Tseytlin, S. S. Eaton, and G. R. Eaton, "A very fast switched-attenuator circuit for microwave and RF applications," Concepts Magn. Reson., Part B 37B, 39-44 (2010).

${ }^{34}$ B. Epel, I. Gromov, S. Stoll, A. Schweiger, and D. Goldfarb, "Spectrometer manager: A versatile control software for pulse EPR spectrometers," Concepts Magn. Reson., Part B 26B, 36-45 (2005). 
${ }^{35}$ B. Epel, V. S. Subramanian, and H. J. Halpem, "Retractable loop-gap resonators for electron paramagnetic resonance imaging with in-situ irradiation capabilities," J. Magn. Reson. (submitted).

${ }^{36}$ C. R. Haney, A. D. Parasca, X. B. Fan, R. M. Bell, M. A. Zamora, G. S. Karczmar, H. J. Mauceri, H. J. Halpern, R. R. Weichselbaum, and C. A. Pelizzari, "Characterization of response to radiation mediated gene therapy by means of multimodality imaging," Magn. Reson. Med. 62, 348-356 (2009).

${ }^{37}$ D. S. Vikram, J. L. Zweier, and P. Kuppusamy, "Methods for noninvasive imaging of tissue hypoxia," Antioxid. Redox Signal. 9, 1745-1756 (2007).

${ }^{38}$ R. D. Braun, J. L. Lanzen, and M. W. Dewhirst, "Fourier analysis of fluctuations of oxygen tension and blood flow in R3230Ac tumors and muscle in rats," Am. J. Physiol. 277, H551-H568 (1999).

${ }^{39}$ M. W. Dewhirst, H. Kimura, S. W. E. Rehmus, R. D. Braun, D. Papahadjopoulos, K. Hong, and T. W. Secomb, "Microvascular studies on the origins of perfusion-limited hypoxia," Br. J. Cancer 74, S247-S251
(1996).

${ }^{40}$ J. Lanzen, R. D. Braun, B. Klitzman, D. Brizel, T. W. Secomb, and M. W. Dewhirst, "Direct demonstration of instabilities in oxygen concentrations within the extravascular compartment of an experimental tumor," Cancer Res. 66, 2219-2223 (2006).

${ }^{41}$ B. H. Robinson, C. Mailer, and A. W. Reese, "Linewidth analysis of spin labels in liquids-I. Theory and data analysis," J. Magn. Reson. 138, 199-209 (1999).

${ }^{42}$ K. Matsumoto, S. English, J. Yoo, K. Yamada, N. Devasahayam, J. A. Cook, J. B. Mitchell, S. Subramanian, and M. C. Krishna, "Pharmacokinetics of a triarylmethyl-type paramagnetic spin probe used in EPR oximetry," Magn. Reson. Med. 52, 885-892 (2004).

${ }^{43}$ G. A. Rinard, R. W. Quine, S. Eaton, and G. R. Eaton, "Frequency dependence of EPR signal intensity, $250 \mathrm{MHz}$ to $9.1 \mathrm{GHz}$," J. Magn. Reson. 156, 113-121 (2002).

${ }^{44}$ X. Pan, D. Xia, and H. Halpern, "Targeted-ROI imaging in electron paramagnetic resonance imaging," J. Magn. Reson. 187, 66-77 (2007). 